\title{
LE DEVELOPPEMENT D'UNE ECHELLE D'ATTITUDE ENVERS LES FEMMES VIOLENTEES PAR LEURS CONJOINTS
}

\author{
FRANCINE LAVOIE, \\ GENEVIEVE MARTIN, \\ et \\ CLAUDE VALIQUETTE \\ Université Laval
}

\begin{abstract}
RESUME
Les personnes concernées par la violence conjugale rapportent que les attitudes prévalant dans la socièté face à ce type de violence sont la source de bien des problèmes. Le manque d'instrument de mesure des attitudes a toutefois limité les recherches. Cet article décrit les étapes suivies dans le développement et la validation d'une echelle d'attitude envers la femme violentée par son conjoint. L'échelle présente des qualités métrologiques suffisantes; elle n'est pas sensible au biais de la désirabilité sociale ni à celui de l'appartenance à un sexe. Comme postulé, une attitude favorable envers la femme violentée est reliée à une attitude non traditionnelle envers les rôles sexuels.
\end{abstract}

Le silence qui a entouré le problème de la violence conjugale pendant de nombreuses années est en soì un phẻnomène difficile à comprendre. Le fait de taire socialement l'existence de ce type de violence a mené à une nonreconnaissance du problème par les divers organismes et intervenant(e)s chargés d'aider les personnes en difficulté. Des femmes victimes de violence de la part de leur conjoint n'ont pas obtenu le support nécessaire. Rosewater (1985) mentionne que des spécialistes de la santé mentale attribuaient à des femmes violentées des diagnostics de dépression et même de schizophrénie, ce sans les avoir interrogées sur l'éventualité de gestes de violence. Stark, Flitcraft, et Frazier (1979) constatent en outre que seulement $2,8 \%$ des dossiers à l'urgence d'un hôpital américain rapportaient la mention "femme violentée" alors qu'une recension de ces dossiers indiquait qu'au moins $10 \%$ devraient l'inclure. Un tel silence est porteur de mépris envers ces femmes.

Or cette non-reconnaissance des problèmes de la femme violentée de la part des organismes et des intervenant(e)s semble refléter celle de la société en général.

Cette recherche a été mence grâce à la subvention du Conseil de Recherches en Sciences Humaines (410-85-1212) accordêe à Geneviève Martin, Francine Lavoie, et Alice Home.

Nous tenons a remercier Claude Gagnon, Patricia Dumas, Anne Chevarie, Martine Gaudreault, et Marie-Christine Pacaud qui ont vu à la passation des questionnaires ainsi qu'Aline Vezina, Les tires-â. part petrent être obtenus aupres du premier auteur: Francine Lavoic, Ecole de psychologie, Université Laval, Citêe universitaire, Quebes, PQ, GIK 7P4, 
Bien qu'un nombre élevé de personnes réprouvent la violence tant physique que psychologique, il n'en reste pas moins que plusieurs dictons et opinions défavorables concernant les femmes violentées par leur conjoint circulent dans la population. Ainsi, des gens émettent l'idée qu'il est compréhensible et même normal qu'un homme batte sa femme ou qu'une femme violentée se mérite la violence, d'autres concluent à la nécessité des chicanes de ménage. Comme on peut le constater, le problème de la violence conjugale suscite des points de vue fort variés.

Les divers points de vue ou opinions reflétant une attitude défavorable envers les femmes violentées peuvent faire en sorte que les victimes camouflent la violence vécue au sein de leur couple et peuvent inciter des hommes à répéter les gestes de violence. En outre, l'entourage de ces femmes et les intervenant(e)s qu'elles consultent peuvent être incités à ne pas appuyer les victimes. A l'opposé, des attitudes désapprouvant la violence peuvent encourager les victimes à faire à temps des demandes d'aide ou encore peuvent amener les personnes violentes à corriger leur comportement. Il semble donc primordial de conduire des études systematiques sur les attitudes face à la femme violentee par son conjoint afin de mieux comprendre les réactions des victimes, des agresseurs, de la population générale, ainsi que celles des intervenant(c)s.

Il existe deux approches dans l'étude des attitudes en rapport avec la violence conjugale: l'enquête sur les attitudes et l'enquête sur l'attribution de la gravité.

Trois recherches sont les plus fréquemment citées comme source d'information sur les attitudes par rapport à la violence conjugale: Greenblat (1983), Stark et Mc Evoy (1970), ainsi que Strauss, Gelles, et Steinmetz (1980). Dans un article où elle fait le point sur ces trois enquêtes, Greenblat (1980) conclut que la majorité ( $75 \%$ et plus) des gens désapprouvent le recours à la force physique entre conjoints. Les hommes et les jeunes condamneraient toutefois moins cette forme de violence. Voulant saisir davantage les justifications ou les conditions jugées appropriées par ceux et celles qui approuvent la violence, Greenblat (1985) note que les justifications les plus souvent évoquées dans les cas de violence envers la femme sont: l'auto-défense, la défense d'un enfant, I'infidélité, ou des comportements hystériques de la part de la femme. Gentemann (1984) montre pour sa part que de tels raisonnements ne sont pas le propre des hommes: une femme sur cinq rapporte au moins une circonstance où il serait justifié pour l'homme de battre sa femme.

Une deuxième approche fournit une appréciation de la gravité accordée au problème de la violence conjugale. Rossi, Waite, Bose, et Berk (1974) ont demandé à des citoyens américains d'ordonner une série de crimes par ordre de gravité. Systématiquement, les violences perpétrées contre l'époux(se) sont perçues comme moins graves. Ainsi, le viol d'une étrangère arrive au quatrième rang en terme de gravité alors que celui de l'épouse arrive au $64^{*}$ rang après le vol par effraction et que le fait de battre son épouse arrive au $94^{\circ}$ rang. Plus récemment, Ursel et Farough (1986) indiquent que des manitobains ordonnant certains crimes selon l'importance du tort causé citent en premier la violence envers les enfants, la conduite en état d'ébriété, puis la violence faite à l'épouse. Les crimes contre la propriété sont jugés moins sérieux. Toutefois, cette province venait de voter une loi dènonçant la violence conjugale. Plus de 10 ans séparent ces deux 
enquêtes, ce qui peut aussi expliquer les différences observées, quoique seule la reprise de l'étude de Rossi et al. (1974) pourrait montrer s'il y a eu évolution des mentalités, Mais de telles études, si intéressantes soient-elles à cause des comparaisons qu'elles permettent, ne donnent toutefois pas l'occasion aux répondant(e)s d'exprimer l'opinion que la violence conjugale n'est pas un crime.

Toutes ces enquêtes ne fournissent donc qu'un portrait partiel de l'attitude envers la violence conjugale. En effet, ne poser qu'une seule question sur le fait de trouver acceptable de frapper ou de gifler sa femme comme le font les trois enquêtes mentionnées ou encore demander de comparer la violence conjugale (violer ou battre) avec d'autres crimes mène à en négliger plusieurs facettes. Car l'attitude des gens envers la femme violentée réfère à de multiples composantes dont les caractéristiques attribuées à ces femmes, les justifications sur les raisons de la violence, et les intentions d'agir face à un tel couple.

C'est pourquoi quelques auteurs ont tenté de développer des mesures d'attitude incorporant plusieurs dimensions. Finn (1986) propose une echelle de cinq items portant sur la légitimité de l'utilisation de la force par l'homme contre sa femme. Les items ne sont cependant pas connus. Le petit nombre d'items porte à croire que l'univers du construit n'est pas couvert adéquatement et cela laisse présager que la consistance interne est relativement faible. Plus intéressante à cause de ses qualités métrologiques de fidélité et de validité est la proposition de Saunders, Lynch, Grayson, et Linz (1987) d'une échelle d'attitude et de croyances face au fait de battre sa conjointe. Cette échelle de 31 items se limite á la violence physique ou plutôt au fait de battre sa conjointe, qui est défini dans les instructions jointes au questionnaire comme frapper de façon répétèe afin d'infliger de la douleur. Les auteurs ne considèrent pas du tout la violence morale ou verbale ou encore la violence générale. De plus, l'échelle ne concerne que les conjoints mariés.

Nous décrirons dans cet article le développement d'une première échelle d'attitude portant sur la violence générale tentant de considérer la violence physique ainsi que la violence morale et verbale. La violence physique référera aux situations où la femme est assaillie sexuellement ou battue par son conjoint, On entendra par violence verbale les incidents oú le conjoint fait des menaces ou du chantage, et par violence morale les incidents où la femme est systématiquement dévalorisée ou isolée. Il s'agit done de femmes violentées par leur conjoint et pas uniquement de femmes battues. De plus, l'échelle concerne autant les conjoints mariés que les conjoints de fait.

\section{DEVELOPPEMENT DE L'ECHELLE D'ATTIIUDE ENVERS LES FEMMES VIOLENTEES PAR LEURS CONJOINTS (AFEVIC)}

L'échelle AFEVIC (Attitude envers les Fermmes Violentées par leurs Conjoints) est une échelle d'attitude dans le sens où seuls sont retenus les items amenant le (la) répondant(e) à poser un jugement de valeur favorable ou défavorable envers la femme qui vit de la violence conjugale ou encore à préciser une intention de comportement favorable ou défavorable à son égard. La théorie des trois composantes de l'attitude (l'affectif, le cognitif, et le conatif ou le com- 
portemental [Eiser, 1979]) inspire notre démarche. L'affectif réfère ici à un sentiment positif ou négatif ou à un souhait, le cognitif à une connaissance et le conatif à une ligne de conduite envers l'objet de l'attitude. Par ailleurs, tout énoncé faisant appel aux connaissances du répondant se trouve exclu de l'échelle, par exemple: "Une canadienne sur 10 a déjà connu de la violence conjugale." De plus, le modèle linéaire de Likert (1932) sert de guide principal dans la construction de rèchelle.

\section{Elaboration des items}

Afin de bien intégrer les diverses facettes du concept au questionnaire, nous avons déterminé à l'aide des principaux ouvrages dans le domaine (entre autres: Carrier \& Michaud, 1982; Greenblat, 1980; Larouche, 1985; Okun, 1986; Stark \& McEvoy, 1970; Strauss et al., 1980) 14 dimensions représentant ce qu'est l'attitude favorable envers la femme violentée par son conjoint. Ainsi, un individu ayant une attitude favorable serait d'accord avec le fait de (a) dénoncer toute forme de violence (autant la violence physique, que verbale ou que morale), (b) n'accepter aucune justification de la violence conjugale, (c) ne pas attribuer la responsabilité à la femme, (d) ne pas favoriser la survie du couple ou de la famille au détriment de la femme violentée, (e) donner foi aux propos de la femme violentée, (f) écouter la femme violentée, (g) s'impliquer personnellement pour lui venir en aide, (h) accepter qu'une remise en question du couple vivant de tels problèmes prenne un certain temps, (i) comprendre et accepter les réactions d'ambivalence, (j) accepter que les professionnel(le)s s'enquièrent automatiquement auprès de leurs clients de possibilité de violence, $(\mathrm{k})$ appuyer les ressources consacrées aux femmes violentées, (1) favoriser des punitions plus sévères pour les conjoints violents, $(m)$ considérer que la violence conjugale est un problème social, (n) croire que la situation puisse être changéc.

Cent cinquante items s'articulant sur ces 14 dimensions furent génèrés à partir de consultations, de lectures, et de discussions au sein de cours. Une première analyse permit de retirer les items concernant les caractéristiques individuelles de l'homme (ex: les hommes violents sont immatures) et les items reliés à des connaissances.

Toutes les dimensions mentionnées sont représentées dans la première version du questionnaire comprenant 61 énoncés. Certaines règles de base (DunnRankin, 1983) ont guidé la rédaction des énoncés: (a) chaque item ne devait contenir qu'une seule idée, (b) l'item ne devait pas être long (20 mots), (c) les expressions "toujours" et "jamais" étaient proscrites, (d) les doubles négations étaient évitées, et (e) les énoncés très polarisès où tout le monde serait en accord ou en désaccord étaient rejetés. II y a également autant d'énoncés favorables que défavorables (Aiken, 1982) une formulation affirmative alternant avec une formulation négative.

Les ètapes suivantes de la construction de l'échelle ont intégré diverses études de validité.

\section{Etude 1: Validation de contenu par la méthode des juges}

La validation de contenu d'une échelle d'attitude permet de vérifier si toutes les dimensions du concept à l'étude se retrouvent dans l'échelle. Il s'agissait donc, 
en plus d'identifier les dimensions non couvertes, de vêrifier si les items référaient vraiment à des attitudes, et de déterminer quels items représentaient une attitude favorable ou défavorable.

La première méthode retenue fut la consultation de quatre expertes dans le domaine qui ont dô émettre par écrit un jugement sur chacun des items (Gable, 1986). Une entente parfaite quant à la valeur favorable ou défavorable et un accord de 3 juges sur 4 a l'effet que l'énoncé décrit une attitude, permettaient de conserver l'item. Sept énoncés ont été rejetés parce qu'ils ne mesuraient pas une attitude et quatre autres à cause d'un manque d'unanimitè sur l'aspect favorable. Aucune autre dimension n'ayant été suggérée, l'échelle fut donc réduite à 50 items.

La deuxième méthode de validation de contenu fut la consultation par entrevue de 10 personnes ayant de 21 a 60 ans provenant de la population générale. Leurs occupations étaient variées: secrétaire, artiste, serveuse de table, écrivain, agent de relations publiques. Certains vivaient même des problèmes de violence conjugale. Trois items ont été rejetés à cause de l'ambiguité quant à laspect favorable. Le vocabulaire de certains items fut revu afin de le simplifier.

Ces dernières personnes furent de plus interrogées quant au mode de réponse pertinent. Le mode de réponse en quatre points fut préféré à celui en deux ou en six points (Gable, 1986). Les réponses sont codifiées de 1 à 4 , de tout à fait d'accord à tout à fait en désaccord.

L'échelle comprenait donc à la fin de cette étude 47 énoncés présentés sous forme d'un test papier-crayon. L'ordre d'apparition des items a été déterminé selon le hasard contrôlé afin d'assurer une répartition égale des ênoncés défavorables et favorables, affirmatifs et négatifs. La cote globale a l'échelle est obtenue en additionnant les scores aux énoncés défavorables et les scores inversés aux énoncés favorables de façon à ce qu'une cote globale èlevée indique une attitude favorable.

\section{Etude 2: Validation de construit}

Cette étude avait comme but de voir si les variations dans les réponses reflètent bien le construit à l'étude et non pas une autre variable, comme par exemple, l'appartenance à un sexe. Ainsi voulant construire une échelle par rapport aux femmes violentèes et non pas une échelle permettant de distinguer les répondants hommes des répondantes femmes, il est important de retirer les items différenciant de façon systématique les hommes des femmes. Par ailleurs, cette étude vise également à s'assurer que les énoncés retenus se rapportent bien au construit principal.

L'échelle AFEVIC a été administrée à 185 étudiant(e)s universitaires de premier cycle de facultés non reliées aux sciences humaines. Comme le rapporte le tableau 1, l'âge moyen de ces répondant(e)s est de 26 ans et il y a un nombre à peu près égal d'hommes et de femmes. A cette étape de l'élaboration de l'échelle, la consistance interne, mesuree par l'alpha de Cronbach est de ,92.

Dix items furent retirés et sept furent modifiés à la suite de cette étude. Les critères d'analyse critique sont en plus de la pertinence théorique, la corrélation item cote globale corrigée (pour l'inclusion de l'item), la variance, et la corréla- 


\section{TABLEAU 1}

\section{Données socio-démographiques des trois} groupes de répondant(e)s

\begin{tabular}{|c|c|c|c|c|c|c|}
\hline \multirow[t]{2}{*}{ Groupe } & \multicolumn{3}{|c|}{ Sexe. } & \multicolumn{3}{|c|}{ Age } \\
\hline & H & Total & F & Moyenne & Ecart-type & Etendue \\
\hline & & 185 & & & & \\
\hline Etudiant(e)s & 90 & & 94 & 26 & 5,43 & $18-46$ \\
\hline & & 471 & & & & \\
\hline Population 1 & 219 & & 249 & 35 & 13,05 & $18-86$ \\
\hline Population? & 67 & 129 & 62 & 32 & 13,65 & $18-70$ \\
\hline $\begin{array}{l}\text { IEtude } 3 \\
{ }^{2} \text { Etude } 4\end{array}$ & & & & & & \\
\hline
\end{tabular}

tion avec le sexe. Les modifications apportées visaient à augmenter la variance. L'echelle contenait done 37 items à la fin de cette étude.

\section{Etude 3: Validation de construit auprès de la population visée}

Cette étude vise à raffiner l'échelle en reprenant les analyses antérieures auprès de la population générale à qui on destine l'échelle (Gable, 1986). Elle vise également á vérifier si l'echelle mesure bien le construit à l'étude et non pas l'effet de la désirabilité sociale.

Quatre cent soixante et onze personnes ont été contactées dans trois centres d'achat, dans une cafétéria pour travailleur(euse)s de centre hospitalier, et dans un centre communautaire pour personnes ágées de la région métropolitaine de Québec. Le nombre de sujets répond au critère de cinq à 10 fois le nombre d'items proposé par Nunally (1967). Le tableau 1 indique que les répondant(e)s ont entre 18 et 86 ans et que l'âge moyen est de 35 ans. Les femmes sont quelque peu plus nombreuses mais selon la même proportion que la population recensée en 1981. Les jeunes sont cependant sur-échantillonnés ( $27 \%$ vs $15 \%$ au recensement) alors que les personnes ágées sont sous-représentées ( $5 \%$ vs $12 \%$ au recensement) les autres groupes d'âge étant mieux représentés. A cette étape, la consistance interne mesurée par l'alpha de Cronbach est de ,85.

Le questionnaire de 33 items de Crowne et Marlowe (1960) a été retenu comme mesure de la désirabilité sociale. Les sujets y répondaient à l'occasion de la passation de l'AFEVIC. Malgré ses lacunes (Nederhof, 1985), cette échelle obtient un haut seuil de stabilité $(\mathrm{r}=, 89)$ et de consistance interne $(\alpha=88)$ (Crowne \& Marlowe, 1964). Une cote élevée indique que le sujet tend à nier avoir des traits indésirables et à ne présenter que ce qui est perçu comme bien vu.

Les mêmes critères qu'à l'étude 2 servent à l'analyse des items tout en ajoutant le retrait éventuel des items corrélés avec la cote globale au Crowne et Marlowe (1960). Six items sont retirés, en particulier à cause d'une faible corrélation avec la cote globale à l'AFEVIC, à cause d'une correlation moyennement 
TABLEAU 2

Détail des résultats pour les 31 items

de l'échelle finale AFEVIC

$\begin{array}{ccc}\begin{array}{l}\text { Sens des Moyenne Variance } \\ \text { items }\end{array} & \begin{array}{c}\text { Corr. } \\ \text { Sexe Corr. } \\ \text { Désirab. } \\ \text { soc. }\end{array} & \text { i-t }\end{array}$

1. On ne peut pas parler de violence lorsqu'il $y$ a une seule agression par le conjoint

$\begin{array}{llllll}- & 3,2 & .71 & -, 01 & -, 08 & .27\end{array}$

2. Une femme qui se fait engueuler par son conjoint est victime de violence conjugale

$\begin{array}{llll}2,9 & , 77 & -, 09 & -, 00\end{array}$

3. Il faut encourager fortement une femme victime de violence conjugale â faire des démarches pour s'en sortir

4. Aucune circonstance ne peut justifier I'homme d'exiger par la force un rapport sexuel de sa conjointe $+$

.64

, 02

$-, 02$

, 38

5. C'est la femme qui amène l'homme à être violent

$-$

3,6

, 44

.20

, 08 .55

6. Une femme devrait tout faire pour maintenir son couple mème si elle est maltraitéc par son conjoint

7. Je contribuerais à une levée de fonds pour subventionner une maison d'hébergement reconnue pour femmes battues

8. Si une femme ne se défend pas quand elle se fait battre par son conjoint, e'est qu'elle aime ça 
9. Faire intervenir la loi dans des chicanes de ménage c'est grossir les problèmes

- 3,2

10. Il faut soutenir une femme violentée qui cherche de l'aide.

11. Une femme qui se fait violenter par son conjoint doit s'arranger toute seule avec son problème

12. Un peu de brutalité dans une relation de couple ne fait pas de mal à la femme

3,7

3,2

.66

.14

,$\infty 0$

, 45 joint

- 3,

3,5

, 45

, 17

, 04
13. Il est inutile d'essayer d'aider une femme violentêe quî refuse de quitter son con-

Une femme devrait faire une plainte officielle contre son conjoint même si elle risque de briser sa famille

\begin{tabular}{|c|c|c|c|}
\hline 3,1 & .65 & ,08 &, 13 \\
\hline 3.2 & .64 & .08 & .16 \\
\hline
\end{tabular}

16. Il serait de mon devoir de citoyen d'intervenir si j'ètais témoin d'un acte de violence entre conjoints

17. Une femme ayant des enfants en bas âge ne devrait pas quitter son conjoint violent

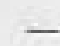

18. Ca sert à rien de porter plainte contre son conjoint violent parce que c'est difficile à prouver

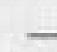

$3,4 \quad, 5$

.04

19. Les chicanes de mènage sont une bonne occasion de rapprochement 
20. La loi devrait punir les hommes qui sont violents envers leurs conjointes $+$

21. La femme violentée a tendance à exagérer la gravité des gestes de son conjoint

\begin{tabular}{|c|c|c|c|c|}
\hline- & 3,1 & .48 &, 19 &, 07 \\
\hline- & 3,2 & .51 & 18 &, 05 \\
\hline+ & 3,1 & .46 & 17 & .09 \\
\hline
\end{tabular}

24. II n'y a pas grand chose a faire pour ameliorer la situation de la femme qui a un conjoint violent

$\begin{array}{llllll}- & 3,4 & .56 & .09 & .17\end{array}$

25. Rien ne peut justifier l'emploi de la violence envers sa conjointe

26. Les femmes n'apprécient pas la rudesse lorsqu'on leur fait l'amour

27. C'est la faute de la femme si elle se fait violer par son conjoint

$3,2 \quad 1,05$ $-, 03 \quad-, 01$

Une femme violentée quí ne fait tien pour s'en sortir est responsable de cette violence $-\quad 2,7$

$2,7 \quad .83$

, 10

.04

29. Je ne me mêlerais pas d'une scène de violence entre conjoints.

30. Une femme devrait avoir honte de dire qu'elle se fait battre

$-\quad 3,6$

3,6

, 41

.12

, 06

31. If faut dénoncer toute forme de violence conjugale

$a=, 88$ 
élevée avec le sexe mais aucun ne fut retiré à cause d'un lien avec le Crowne et Marlowe (1960). Le médiane des corrêlations des items avec le Crowne et Marlowe (1960) était de $r=, 06$.

Le tableau 2 indique le détail de l'analyse pour chacun des 31 items retenus dans l'échelle finale, alors que le tableau 3 montre que les proprićtés de l'échelle sont quelque peu semblables mais non identiques pour les deux sexes. La moyenne pour l'ensemble des répondant(e)s de la population gènérale est de 102 et l'écart-type de 11,07. La moitié des corrélations item et cote globale se situent en haut de ,43. La corrélation entre la cote globale de l'AFEVIC et celle de l'échelle de Crowne et Marlowe (1960) est relativement faible $(r=, 12)$. Il en est de même pour la corrélation avec le sexe $(r=, 21)$. La consistance interne est de , 88 ce qui est suffisamment élevé. Cette version finale de 31 items intégre 11 des 14 dimensions citées come décrivant une attitude favorable. Les dimensions suivantes se trouvent exclues à la suite des analyses: écouter la femme violentée (six), accepter que les professionnel(le)s s'enquièrent automatiquement auprès de leurs clients de possibilité de violence (10), considérer que la violence conjugale est un problème social (13). L'échelle couvre donc bien le concept théorique à l'étude.

\section{Etude 4: Validation de construit avec un construit théoriquement reliế}

Une autre modalité d'étude de la validité d'une échelle consiste en la vérification empirique de la relation entre le construit mesuré par cette échelle et un autre construit relié théoriquement pour lequel il existe une mesure validée (Carmines \& Zeller, 1979). Plusieurs auteurs (Coleman, 1980; Strauss, 1980; Strauss et al., 1980) ayant discutè du lien entre une attitude traditionnelle face aux rôles sexuels et une attitude favorable ou de tolérance à la violence conjugale, il fut décidé d'étudier cette relation. D'ailleurs trois recherches ont rapporté une telle relation. Gentemann (1984) conclut que des femmes plus conservatrices justifient davantage la violence conjugale faite aux femmes (gamma $=-, 50$ ). Finn (1986) rapporte l'existence d'une relation modérément élevée $(\mathrm{r}=, 65 p<.01)$ indiquant que des étudiant(e)s de niveau collégial endossant des valeurs traditionnelles face aux rôles sexuels approuvent davantage le recours par l'homme à la force

\section{TABLEAU 3}

\section{Caractéristiques de l'échelle AFEVIC finale} de 31 items chez la population généralet selon le sexe

\begin{tabular}{ccccccc}
\hline Sexe & N & $\bar{X}$ & $\sigma$ & $\alpha$ & Md (nit) & rem \\
\hline H & 211 & 99,76 & 11,83 &, 90 & .45 & .15 \\
F & 224 & 104,30 & 9,84 &, 85 &, 38 &, 03 \\
H + F & 435 & 102,10 & 11,07 &, 88 & .43 &, 12 \\
\hline
\end{tabular}

riexe, 21

Tableau 1, population 1 
physique face à son épouse. Enfin, Saunders et al. (1987) utilisant le questionnaire de Spence, Helmreich, et Stapp (1973) pour valider leur échelle sur la violence trouvent que les gens traditionnels endossent les croyances qu'il est justifié de battre sa femme, que la femme tire profit de cette violence physique, et qu'on ne devrait pas aider ces femmes. Il semble donc bien y avoir un lien entre ces deux attitudes ce qui justifie le recours à une échelle d'attitude envers les rôles sexuels afin de valider AFEVIC.

L'échelle utilisèe est la version abrégée de 15 items de l'échelle d'attitude envers les femmes (Attitudes Toward Women [AWS]) de Spence et Helmreich (1978) qui mesure l'attitude envers les droits et les rôles des femmes. Cette échelle abrégée présente une corrélation de, 91 avec l'échelle originale et un coefficient alpha de ,89. L'échelle de 25 items présentait des qualités métrologiques suffisantes. Spence et al. (1973) rapportent qu'elle distingue bien les hommes des femmes alors que Kilpatrick et Smith (1974) montrent que les membres d'un groupe féministe obtiennent un score nettement plus élevé et que Stanley, Boots, et Johnson (1975) concluent que des groupes critères se distinguent bien par leur cote.

Un nouvel échantillon de 129 personnes recrutées dans deux centres d'achat a participé à cette dernière étude. Leur âge varie de 18 à 70 ans et l'äge moyen est de 32 ans (tableau 1 ).

La corrélation de Pearson entre le score à l'AFEVIC et le score au AWS est de ,68 $(p<.01)$. Cela indique qu'il $y$ a un lien modérément élevé entre le fait d'avoir une attitude traditionnelle face aux rôles sexuels et une attitude défavorable envers les femmes violentèes par leurs conjoints. Il ne fallait pas obtenir une corrélation élevée ce qui aurait indiqué que possiblement les deux échelles mesuraient le même construit, Le confirmation d'une relation modérément élevée est un indice de la validité d'AFEVIC.

\section{CONCLUSION}

L'échelle AFEVIC présente les qualités requises pour être utilisée comme indicateur de l'attitude envers les femmes violentées par leurs conjoints mariés ou de fait. Ayant un coefficient de consistance interne de , 88 il semble que le construit qu'elle mesure soit relativement cohérent. De plus, l'échelle est peu influencée par la désirabilité sociale des répondant(e)s et par l'appartenance à un sexe. En fait, cette échelle convient bien aux femmes et aux hommes. Elle est egalement appropriée pour la population adulte générale contrairement à bien des échelles limitées à des populations étudiantes. De plus, cette échelle permet de mesurer l'attitude envers la violence générale et non pas seulement envers le fait de battre sa conjointe.

Lors de la construction d'AFEVIC, plusieurs stratégies ont permis d'assurer la validité de contenu et de construit. Une dernière étape de validité de construit a de plus été menée avec l'échelle finale en vérifiant le lien entre une attitude favorable aux femmes violentées (AFEVIC) et une attitude non-traditionnelle face aux rôles de la femme. D'autres recherches, en particulier celle de la stabilitê test-retest et celle de groupes-critères, permettront de s'assurer davantage de la fiabilité et de la validité de l'échelle. 
L'utilité de l'échelle AFEVIC est de permettre de situer une personne sur un continuum d'évaluation bipolaire face à l'objet d'attitude: la femme violentée par son conjoint. Une personne ayant une attitude favorable d'après l'AFEVIC serait d'accord avec le fait de ne pas attribuer la responsabilité de ce comportement a la femme, de ne pas favoriser la survie du couple ou de la famille au détriment de la femme; elle accepterait qu'on offre de l'aide a la femme. Comme pour toute mesure d'attitude, il faut recourir à l'étude de la cote globale et non pas à l'analyse item par item car les vérifications de la qualité de l'échelle se sont faites à partir de la cote globale.

Parmi les limites d'un tel questionnaire, soulignons que l'auto-évaluation non-anonyme d'un individu à l'aide de l'échelle pourrait amener certaines distorsions à cause de la possibilité de fausser les résultats. Mais il est possible de minimiser ce biais lorsque l'évaluation se déroule anonymement ou dans un climat peu menaçant et favorise plutôt l'atteinte d'une meilleure compréhension de soi.

Il est possible d'entrevoir plusieurs utilisations d'une telle échelle entre autres dans l'évaluation de programmes de formation ou de modification d'attitudes, dans la sensibilisation d'intervenant(e)s ou du grand public. Les intervenant(e)s communautaires impliqué(e)s dans le travail auprès de couples, de familles, et d'associations auront dorénavant accès à un nouvel outil qui pourra se révéler utile dans la prévention et le traitement de la violence conjugale.

\begin{abstract}
Societal attitudes toward conjugal violence are a major source of problems. However, lack of instrumentation has hindered meaningful research. The aim of the present study is to describe the sequential development and subsequent validation of an attitude scale toward women victims of conjugal violence. The final scale demonstrates proper metrological qualities and is free from social desirability and sex bias. As predicted, there is a significant relationship between favourable attitudes toward women victims of violence and toward non-traditional sex roles.
\end{abstract}

\title{
REFERENCES
}

Aiken, L.R. (1982). Psychological testing and assessment (4th. ed.), Boston: Allyn \& Bacon.

Carmines, E.G., \& Zellers, R.A. (1979). Reliability and validity assessment. London: Sage.

Carrier, M., \& Michaud, M. (1982). La violence faite aux femmes en milieu conjugal: Le produit d'une sociéré sexiste. Ottawa: Secrétariat d'Etat.

Coleman, K.H., Weiman, M.L., \& Hsi, B.P. (1980). Factors affecting conjugal violence. The Journal of Psychology, 105, 197-202.

Crowne, D.P., \& Marlowe, D. (1960). A new scale of social desirability of psychopathology. Journal of Consulting Psychology, 24, 349-354.

Crowne, D.P., \& Marlowe, D. (1964). The approval motive: Studies in exaluative dependence, Wiley: New York.

Dunn-Rankin, P. (1983). Scaling methods. London: Lawrence Erlbaum.

Eiser, R.J. (1979). Attitudes. In K. Connoly (Ed,), Psychology survey (2nd ed.). New York: G, Allen. 
Finn, J. (1986). The relationslip between sex role attitudes and attitudes supporting marital violence. Sex Roles, 14, 235-243.

Gable, R.K. (1986). Instrument development in the affective domain. Boston: KluwerNijhoff,

Gentemann, K.M. (1984). Wife beating: attitudes of a non-clinical population. Victimology: An International Journal, 9(1), 109-119.

Greenblat, C.S., (1983). A hit is a hit is a hit ... or is it? Approval and tolerance of the use of physical force by spouses, In D. Finkelhor, R.J. Gelles, G.T. Hotaling, M.A. Strauss (Eds.), The dark side of families. Beverly Hills: Sage.

Greenblat, C.S. (1985), Don't hit your wife ... unless . ... : Preliminary findings on normative support for the use of physical force by husbands, Victimology: An International Journal, 10, 221-241.

Kilpatrick, D.G., \& Smith, A.D. (1974). Validation of the Spence-Helmreich Attitude Toward Women scale. Psychological Reports, 35, 461-462.

Larouche, G. (1985). Guide d'intervention auprès des femmes violentées. Montréal: Corporation des travailleurs sociaux du Québec.

Likert, R. (1932). A technique for the measurement of attitudes. Archives of Psychology, 140-152.

Nederhof, A.J. (1985). Methods of coping with social desirability bias: A review. European Journal of Social Psychology, 15, 263-280.

Nunally, J.C. (1967). Psychometric theory. New York: McGraw-Hill.

Okun, L. (1986). Woman abuse. Facts reptacing myths. New York: State University of New York.

Rosewater, L.B. (1985). Schizophrenic, borderline, or battered? In L.B. Rosewater \& L.E. Walker (Eds.), Handbook of feminist therapy: Women's issues in psychotherapy. New York: Springer.

Rossi, F.E., Waite, C., Bose, E., \& Berk, R. (1974). The seriousness of crimes: Normative structure and individual differences. American Sociological Review, 79, 224-237.

Saunders, D.G., Lynch, A.B., Grayson, M. \& Linz, D. (1987). The inventory of beliefs about wife-beating: The construction and initial validation of a measure of beliefs and attitudes. Violence and Victims, 2, 39-57.

Spence, J.T., \& Helmreich, R.L. (1978). Masculinity and femininity: Their psychological dimensions, correlates and antecedents. Austin: University of Texas Press.

Spence, J.T. Helmreich, R.L., \& Stapp, J. (1973). A short version of the Attitudes Toward Women scale (AWS). Bulletin of the Psychonomic Saciety, 2, 219-220.

Stanley, G., Boots, M., \& Johnson, C. (1975). Some Australian data on the short version of the Attitudes Toward Women scale (AWS). Australian Psychologist, 10, 319-323.

Stark, E., Fliteraft, A, \& Frazier, W. (1979). Medicine and patriarchal violence: The social construction of a private event, International Journal of Health Services, 9(3), 461-489.

Stark, R., \& McEvoy, J. (1970). Middle-class violence. Psychology Today, 4 (52-53), 110-112.

Strauss, M.A. (1980). Wife-beating: How common and why? Victimology: An International Journal, 2, 443-458.

Strauss, M.A., Gelles, R.J., \& Steinmetz, S.K. (1980). Behind closed doors; Violence in the American family. New York: Anchor Books.

Ursel, E.J., \& Farough, D. (1986). The legal and public response to the new wife abuse directive in Manitoba, Canadian Journal of Criminology, 28, 171-184. 